\title{
USO DE ENXERTO ÓSSEO HOMÓLOGO ESTRUTURAL CORTICAL EM CIRURGIAS DE RECONSTRUÇÃO FEMORAL
}

\author{
USE OF CORTICAL STRUCTURAL HOMOLOGOUS BONE GRAFT \\ IN FEMORAL RECONSTRUCTINE SURGERY
}

\author{
Milton Valdomiro Roos ${ }^{1}$, Bruno Dutra Roos ${ }^{2}$, Taís Stedile Busin Giora ${ }^{3}$, Thiago Martins Taglietti ${ }^{3}$
}

\section{RESUMO}

Objetivo: Realizar uma avaliação clínica e radiográfica dos pacientes submetidos a tratamento cirúrgico com utilização de enxerto ósseo homólogo estrutural cortical em cirurgias de reconstrução femoral secundárias a afrouxamento de artroplastia total do quadril e fraturas periprotéticas. Métodos: Foi realizado estudo retrospectivo com 27 pacientes submetidos a tratamento cirúrgico de reconstrução femoral secundária a afrouxamento de artroplastia total de quadril (12 casos) e fratura periprotética (15 casos) utilizando enxerto homólogo estrutural cortical e implante cimentado, no período de junho de 1999 a fevereiro de 2008. Desses, 21 preencheram todos os critérios necessários para este trabalho. Os pacientes foram submetidos a uma avaliação clínica pré e pós-operatória, de acordo com o Harris Hip Score. Foram avaliadas também radiografias pré-operatórias, pós-operatórias imediatas e tardias, comparando a consolidação das fraturas, os sinais radiográficos de consolidação do enxerto, a modificação do estoque ósseo e da qualidade óssea do fêmur e o alinhamento femoral. Resultados: Nove pacientes $(42,9 \%)$ foram submetidos à reconstrução femoral secundária a afrouxamento de artroplastia total de quadril e $12(57,1 \%)$, à reconstrução femoral secundária à fratura periprotética. Com relação à classificação clínica pós-operatória, os resultados obtidos foram considerados como satisfatórios em $85,7 \%$ dos casos e insatisfatórios em 14,3\%. Sinais radiográficos de consolidação do enxerto foram visualizados em todos os casos. Houve aumento do estoque ósseo em $90,5 \%$ das reconstruções de quadril realizadas, conforme aferição do índice cortical. Além disso, a modificação da qualidade óssea femoral foi considerada boa em $66,7 \%$ dos casos. Conclusão: O uso de enxerto ósseo homólogo estrutural cortical em cirurgias de reconstrução femoral de artroplastias totais do quadril e em fraturas periprotéticas é uma boa opção de tratamento em casos selecionados, permitindo resultados clínicos e radiográficos satisfatórios.

Descritores - Fraturas do Fêmur; Artroplastia de Quadril/métodos; Prótese de Quadril; Transplante Ósseo

\section{ABSTRACT}

Objective: To perform a clinical and radiographic assessment of patients submitted to cortical structural homologous bone graftsurgical treatment for femoral reconstruction following mechanical failure of total hip arthroplasty and periprosthetic fractures. Methods: A retrospective study with 27 patients submitted to surgical treatment for femoral reconstruction following mechanical failure of total hip arthroplasty (12 cases) and periprosthetic fractures (15 cases), using cortical structural homologous bone graft and cemented implants, in the period of June 1999 to February 2008 was performed. Of these, 21 fulfilled the criteria required for this study. Patients were submitted to a pre and postoperative clinical assessment according to Harris Hip Score. Pre-operative, immediate and late postoperative radiographs were also evaluated by comparing fracture consolidation, radiographic signs of graft integration, changes in bone stock and femoral bone quality, as well as femoral alignment. Results: Nine patients (42.9\%) were submitted to femoral reconstruction following mechanical failure of total hip arthroplasty. The other 12 cases (57.1\%), were submitted to femoral reconstruction following periprosthetic fracture. Results were considered satisfactory in $85.7 \%$ and unsatisfactory in $14.3 \%$ of cases. Radiographic signs of graft integration to the host's bone were seen in all cases. There was an increase of bone stock in $90.5 \%$ of hip reconstructions, according to cortical index measurement. Furthermore, changes in femoral bone quality were considered good in $66.7 \%$ of cases. Conclusion: The use of cortical structural homologous bone grafts for both femoral reconstructive surgeries of total hip arthroplasty and periprosthetic fractures is a good treatment option for selected cases, allowing for satisfactory clinical and radiographic results.

Keywords - Femoral Fractures; Arthroplasty, Replacement, Hip/methods; Hip Prosthesis; Bone Transplantation

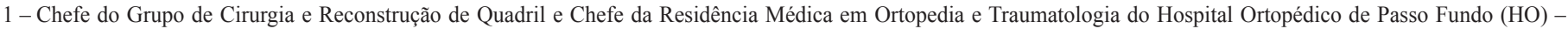
Passo Fundo, RS.

2 - Fellow em Cirurgia do Quadril do Hospital Ortopédico de Passo Fundo (HO) - Passo Fundo, RS.

3 - Médico Residente em Ortopedia e Traumatologia do Hospital Ortopédico de Passo Fundo (HO) - Passo Fundo, RS.

Trabalho realizado no Hospital Ortopédico de Passo Fundo, Faculdade de Medicina da Universidade de Passo Fundo - Passo Fundo, RS, Brasil

Correspondência: Taís Stedile Busin Giora, Av. Sete de Setembro, 817, Centro - 99010-121 - Passo Fundo (RS), Brasil. E-mail: tais@giora.com.br 


\section{INTRODUÇÃO}

A perda de estoque ósseo femoral é uma grave complicação que pode ocorrer tanto em decorrência de fraturas periprotéticas como nos afrouxamentos das $\operatorname{artroplastias~}^{(1,2)}$.

A incidência de fraturas periprotéticas, que compreende $1 \%$ a $6 \%$ das artroplastias de quadril, tem crescido significativamente durante a última década em razão do aumento da longevidade dos pacientes ${ }^{(3,4)}$. Estas fraturas ocorrem mais comumente após artroplastias primárias, decorrentes de dano cortical transoperatório. Por sua vez, aquelas ocorridas durante ou após artroplastias de revisão têm relação com a perda do estoque ósseo femoral $^{(5,6)}$. Nestes casos, o tratamento baseia-se nas condições clínicas do paciente, localização da fratura, estabilidade do componente femoral e na qualidade óssea do terço proximal do fêmur ${ }^{(7)}$.

Há inúmeras técnicas cirúrgicas e implantes desenvolvidos para reconstrução femoral secundária a afrouxamento de artroplastia de quadril, o que evidencia a complexidade do seu tratamento ${ }^{(8,9)}$. Esses casos podem cursar com lesões osteolíticas extensas, descontinuidade óssea e ruptura do implante ${ }^{(10,11)}$.

O objetivo das reconstruções femorais e fixação de fraturas periprotéticas é a obtenção de um implante estável, com manutenção ou reposição do estoque ósseo ${ }^{(12,13)}$. As opções de tratamento incluem hastes cimentadas ou não cimentadas, às quais se pode associar enxertia óssea autóloga ou homóloga ${ }^{(14,15)}$. O uso de enxerto ósseo homólogo estrutural permite altas taxas de consolidação das fraturas e aumento do estoque ósseo femoral ${ }^{(3,16)}$.

O objetivo do presente estudo é avaliar os resultados clínicos e radiográficos da utilização de enxerto ósseo homólogo estrutural cortical em cirurgias de reconstrução femoral secundárias a afrouxamento de artroplastia total do quadril e fraturas periprotéticas.

\section{MÉTODOS}

O presente estudo foi aprovado pelo Comitê de Ética em Pesquisa do Hospital Ortopédico de Passo Fundo.

Foi realizado um estudo retrospectivo com 27 pacientes submetidos a tratamento cirúrgico de reconstrução femoral secundária a afrouxamento de artroplastia total de quadril (12 casos) e fratura periprotética (15 casos) utilizando enxerto ósseo homólogo estrutural cortical (não irradiados) e implante cimentado, no período de junho de 1999 a fevereiro de 2008. Todas as cirurgias foram realizadas pela mesma equipe médica do Serviço de Cirurgia do Quadril do Hospital Ortopédico de Passo Fundo, RS.

Foram incluídos no estudo pacientes com uma ou mais artroplastias prévias que apresentavam perda de estoque ósseo do fêmur secundário a afrouxamento asséptico de artroplastia ou fratura periprotética, os quais foram submetidos a tratamento cirúrgico utilizando enxertia homóloga estrutural cortical, com seguimento mínimo de um ano. Os critérios de exclusão foram: perda do seguimento (quatro casos) e exames radiográficos incompletos (dois casos).

Conforme os critérios estabelecidos, 21 pacientes foram incluídos neste estudo, sendo 17 do sexo feminino (81\%) e quatro do sexo masculino (19\%), com o lado direito sendo o mais acometido (13 fêmures).

A média de idade dos pacientes no momento da cirurgia foi de 62 anos, variando de 35 a 81 anos. O seguimento médio foi de dois anos e 11 meses, sendo mínimo de um ano e máximo de sete anos e três meses.

A avaliação clínica pré e pós-operatória foi realizada em todos os pacientes, objetivando classificá-los pelo Harris Hip Score ${ }^{(17)}$. Consideraram-se como resultados pós-operatórios ruins pacientes com escore menor de 70; razoáveis, entre 70 e 79; bons, entre 80 e 89; e excelentes, entre 90 e 100. Resultados clínicos excelentes e bons foram classificados como satisfatórios. Os resultados razoáveis e ruins foram considerados insatisfatórios ${ }^{(18)}$.

Foi utilizado enxerto ósseo homólogo estrutural cortical ("régua óssea") proveniente do Banco de Tecidos Musculoesqueléticos do Hospital São Vicente de Paulo de Passo Fundo, não irradiado, processado a partir de segmentos de diáfise de fêmur e tíbia de doadores. $\mathrm{O}$ seu comprimento é padronizado em $2,5 \mathrm{~cm}$ de largura por $15 \mathrm{~cm}$ de comprimento, e foram ajustados transoperatoriamente conforme as necessidades cirúrgicas.

Em 19 pacientes (90,5\% dos casos) foi utilizado enxerto ósseo homólogo estrutural associado à amarria por cerclagem como método de estabilização da reconstrução femoral. Destes, em nove casos $(42,9 \%)$ observou-se a presença do enxerto na face lateral e anterior do fêmur e, em 10 casos $(47,6 \%)$, somente na face lateral. Nos dois pacientes restantes $(9,5 \%$ dos casos) foi utilizado enxerto estrutural na face anterior do fêmur associado à amarria por cerclagem e fixação da fratura com placa e parafusos.

Os defeitos ósseos femorais foram determinados conforme a classificação de D'Antônio, adotada pela American Academy of Orthopaedic Surgeons (AAOS) ${ }^{(19)}$. Já as fraturas periprotéticas foram avaliadas de acordo com a classificação de Vancouver apud Masri et al ${ }^{(20)}$. 
Todos os pacientes selecionados para o estudo apresentavam radiografias pré-operatórias e pós-operatórias imediatas e tardias, as quais foram comparadas quanto à consolidação das fraturas, aos sinais radiográficos de consolidação do enxerto estrutural cortical, à modificação do estoque ósseo e da qualidade óssea do fêmur e ao alinhamento femoral.

\section{Análise radiográfica}

A análise radiográfica do enxerto homólogo estrutural cortical foi realizada por dois observadores separadamente, utilizando um mesmo paquímetro e régua, com o objetivo de evitar erros intraobservador. Radiografias de pelve e fêmur nas incidências anteroposterior e perfil foram analisadas em relação à consolidação das fraturas (nos casos de fraturas periprotéticas), sinais radiográficos de consolidação do enxerto, modificação do estoque ósseo e da qualidade óssea do fêmur, e alinhamento femoral (Figuras 1 e 2).
A consolidação das fraturas periprotéticas foi caracterizada pela continuidade da densidade radiográfica e formação de calo ósseo sobre o foco de fratura ${ }^{(3)}$.

Os sinais radiográficos da consolidação do enxerto foram determinados conforme critérios de Emerson et $a l^{(13)}$ em cinco categorias: 1) reabsorção das arestas proximais e distais do enxerto estrutural (correspondendo à suavização dos contornos do enxerto estrutural cortical); 2) presença de erosões ósseas (indicando neovascularização do enxerto), 3 ) ponte óssea parcial; 4) ponte óssea total; 5) obliteração da interface enxerto-osso hospedeiro (indicando revascularização do enxerto); 6) reabsorção (com diminuição da espessura do enxerto ósseo estrutural em grau variado, correspondendo ao remodelamento do fêmur). $\mathrm{O}$ enxerto pode apresentar características de mais de uma categoria simultaneamente, sendo enfatizado pelo autor que a formação de ponte óssea parcial maior que $50 \%$ entre o enxerto homólogo estrutural cortical e o osso
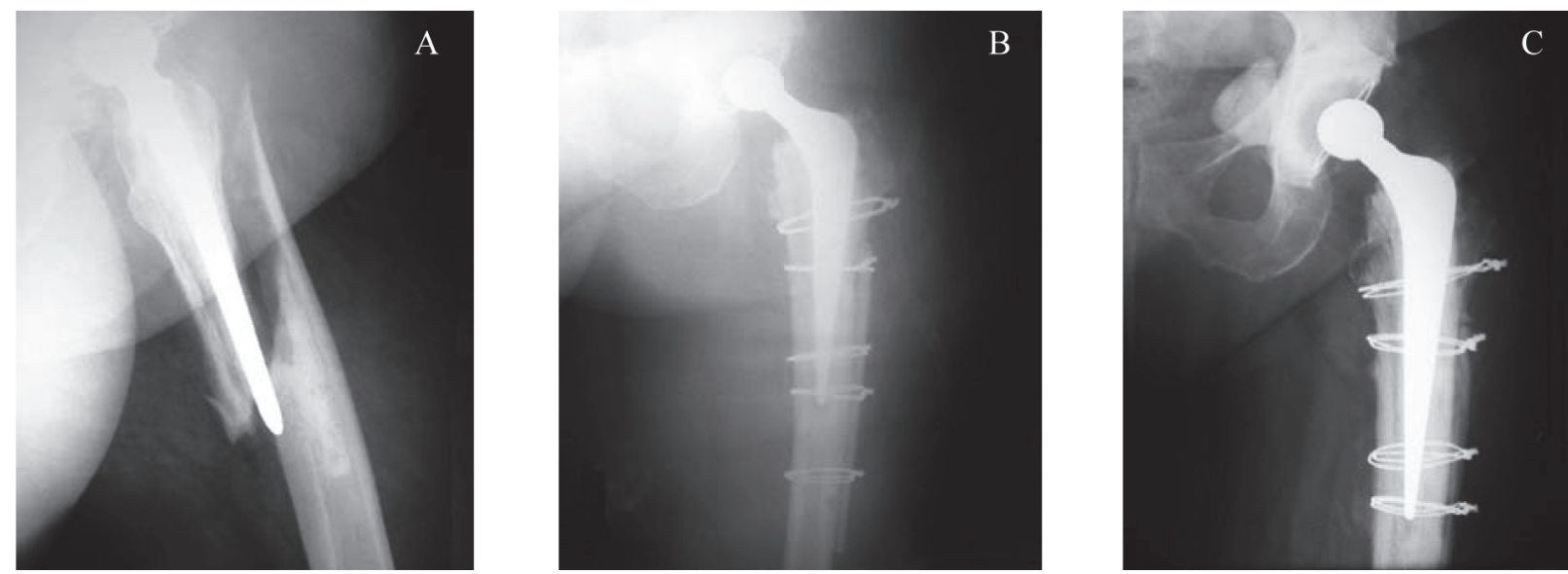

Figura 1 - A) Radiografia pré-operatória de fratura periprotética; B) Radiografia pós-operatória imediata, evidenciando fixação com enxerto ósseo estrutural cortical e amarria por cerclagens; C) Radiografia pós-operatória com seguimento de um ano e sete meses, evidenciando consolidação da fratura e do enxerto ósseo.
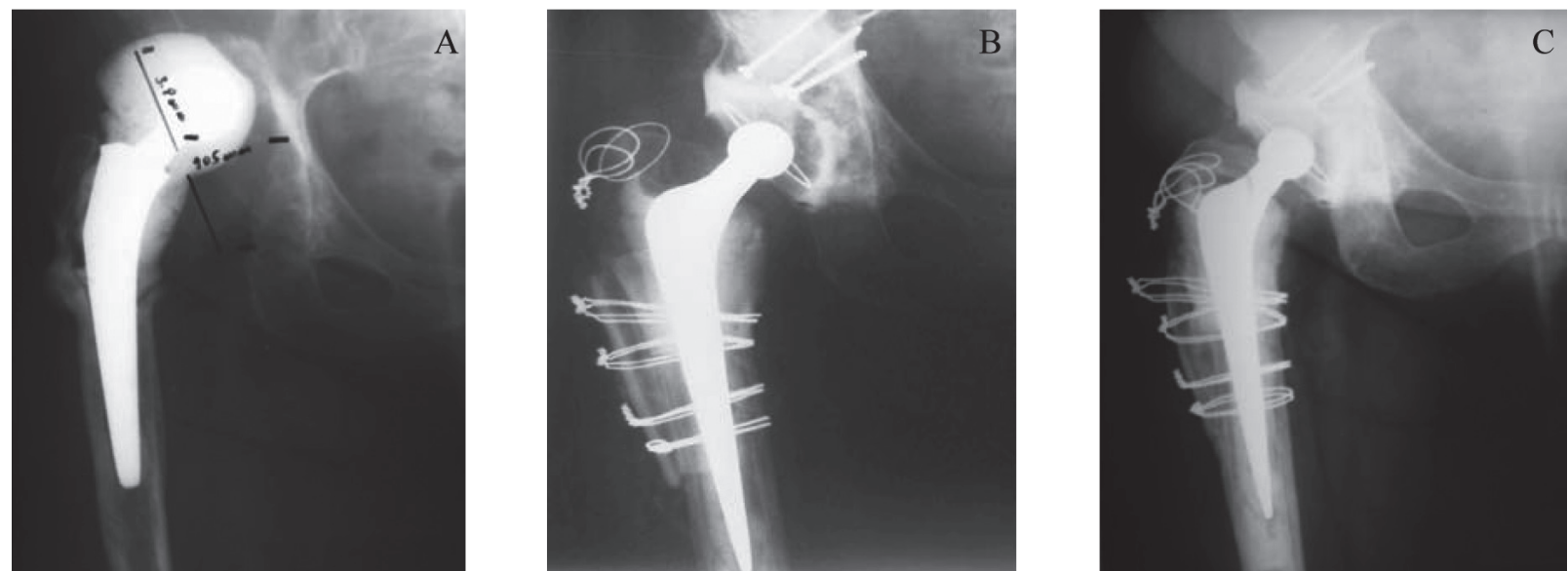

Figura 2 - A) Radiografia pré-operatória de revisão femoral; B) Radiografia pós-operatória imediata, evidenciando a utilização de enxerto ósseo estrutural cortical e amarria por cerclagens; C) Radiografia pós-operatória com seguimento de três anos e 10 meses, evidenciando consolidação do enxerto ósseo. 
hospedeiro ou formação de ponte total são critérios absolutos de consolidação do enxerto.

A modificação do estoque ósseo femoral foi analisada conforme os critérios de Haddad et al ${ }^{(3)}$ através do índice cortical, que corresponde à relação entre o diâmetro femoral e do canal medular, medidos um centímetro distalmente ao pequeno trocanter, que serve para quantificar a perda óssea refletida no istmo femoral. Este índice foi medido em radiografias na incidência anteroposterior e perfil, no pré-operatório e no pós-operatório mais tardio. Esta aferição não pôde ser realizada em dois pacientes tratados com fixação cirúrgica de fraturas periprotéticas utilizando-se placas, pois houve sobreposição da imagem do metal sobre a cortical femoral.

A qualidade óssea do fêmur foi classificada, conforme Callaghan et $a l^{(21)}$, como boa ou ruim, mediante a comparação de radiografias realizadas no pré-operatório e no pós-operatório mais tardio. Considera-se como ruim quando a espessura cortical femoral medida no pós-operatório tardio a $10 \mathrm{~cm}$ do trocanter menor diminuir $50 \%$ em relação ao pré-operatório, ou quando diminuir $75 \%$ a $5 \mathrm{~cm}$ do trocanter menor, ou se ambas as medidas da cortical em qualquer ponto abaixo do trocanter menor medirem menos de $4 \mathrm{~mm}$.

\section{Estabilização do enxerto homólogo estrutural cortical}

Foi utilizado enxerto ósseo homólogo estrutural cortical, posicionado na face lateral e/ou anterior do fêmur, o qual foi fixado por meio de amarria por cerclagem e parafusos. A interface enxerto-osso hospedeiro foi ocupada com enxerto ósseo homólogo fragmentado, objetivando evitar a formação de "espaço morto", que permitiria a formação de tecido fibroso ou cistos, dificultando a consolidação do enxerto estrutural (Figura 3).

\section{Análise estatística}

As comparações entre as medidas radiográficas pré e pós-operatórias com relação à modificação do estoque ósseo e da qualidade óssea femoral foram realizadas utilizando-se o teste não-paramétrico de Wilcoxon signed-ranks, visto ser uma amostra com variância e coeficiente de assimetria variáveis. Significância estatística foi considerada com $p<0,05$. Os dados estatísticos foram calculados com o pacote SPSS 15.0.

\section{RESULTADOS}

Do total de pacientes avaliados, nove (42,9\%) foram submetidos a tratamento cirúrgico de reconstrução femoral secundária a afrouxamento de artroplastia total de quadril e os outros 12 casos $(57,1 \%)$, à fixação cirúrgica de fratura periprotética utilizando enxerto ósseo homólogo estrutural cortical (Figura 4).

A classificação clínica pré-operatória, de acordo com o Harris Hip Score ${ }^{(17,18)}$, foi realizada apenas em nove

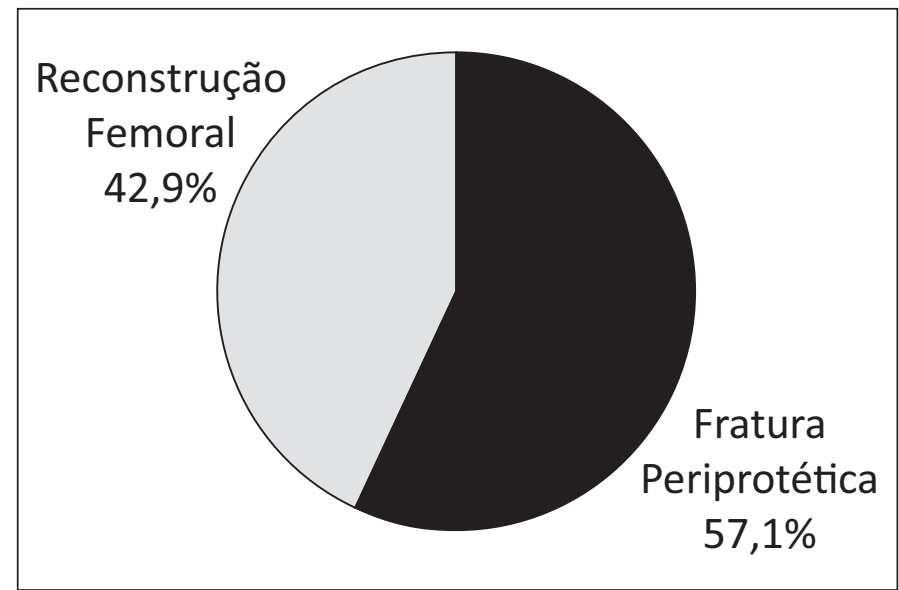

Figura 4 - Caracterização dos pacientes quanto ao procedimento cirúrgico realizado.
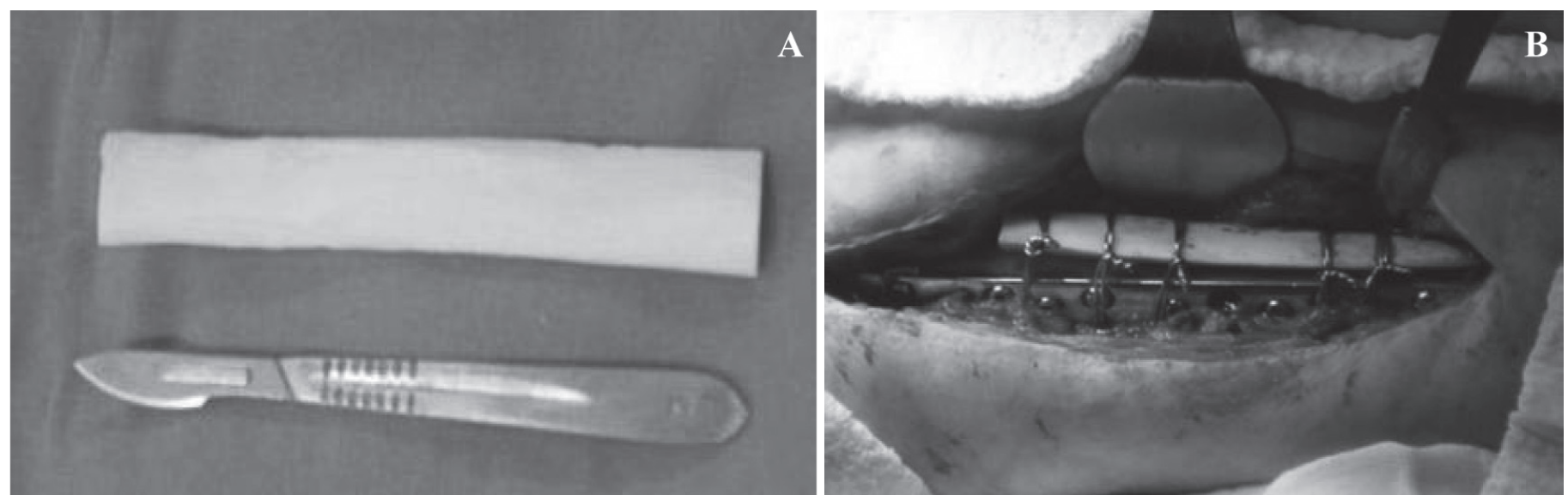

Figura 3 - A) Enxerto ósseo homólogo estrutural cortical; B) Transoperatório com fixação do enxerto por meio de cerclagem. 
pacientes. Nos 12 casos restantes, tal avaliação não foi efetuada em consequência da presença de fratura periprotética. Nos casos avaliados, obteve-se média de 30 pontos (22 a 42 pontos). No pós-operatório, consideraram-se os resultados como excelentes em oito $(38,1 \%)$, bons em $10(47,6 \%)$, razoáveis em dois $(9,5 \%)$ e ruim em um paciente $(4,8 \%)$. Portanto, resultados clínicos satisfatórios foram obtidos em $85,7 \%$ dos casos. A média de pontos foi de 85 (62 a 98 pontos) (Figura 5).

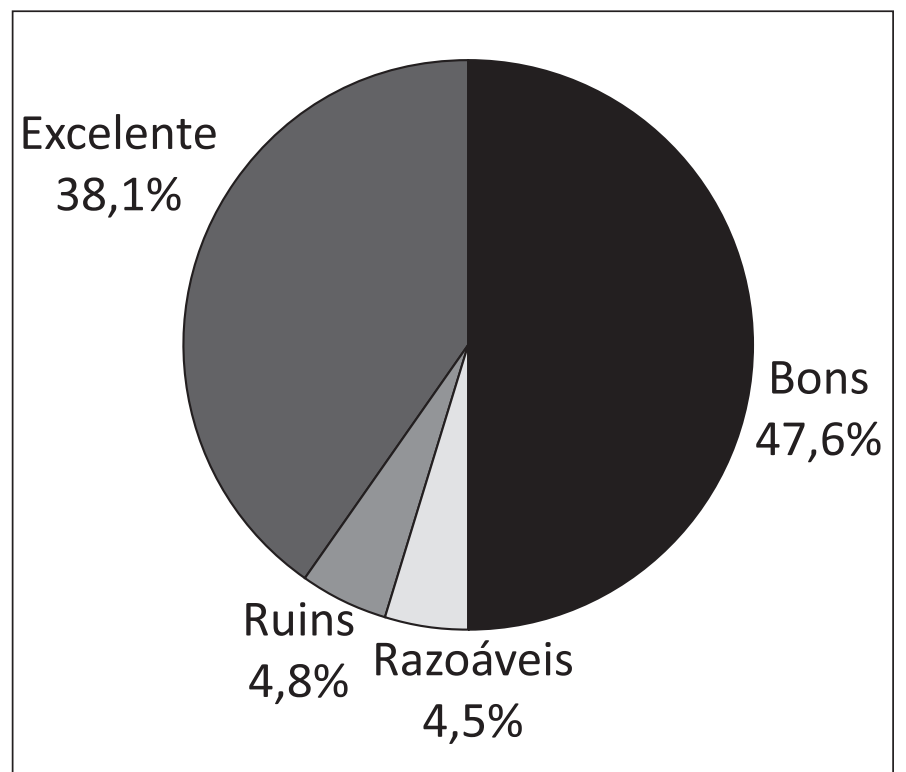

Figura 5 - Classificação clínica pós-operatória de acordo com o Harris Hip Score ${ }^{(17,18)}$.

De acordo com a classificação de D'Antônio, adotada pela $\operatorname{AAOS}^{(19)}$, cinco defeitos femorais $(23,8 \%)$ foram considerados segmentares (tipo I); dois (9,5\%), cavitários (tipo II); dois (9,5\%), combinados (tipo III) e $12(57,2 \%)$, descontinuidade (tipo VI). Todas as fraturas periprotéticas foram classificadas como defeitos ósseos tipo VI (Figura 6).

Com relação à classificação de Vancouver apud Masri et $a l^{(20)}$, para fraturas periprotéticas, um caso foi considerado B1 (8,3\%); três casos, B2 (25,1\%); quatro casos, B3 (33,3\%); e quatro casos, C (33,3\%). Em todos esses pacientes houve consolidação do foco de fratura.

Sinais radiográficos de consolidação do enxerto foram visibilizados em todos os pacientes. Todos os casos apresentaram erosões ósseas. Cinco casos $(23,8 \%)$ obtiveram formação de ponte parcial maior que $50 \%$ da extensão do enxerto estrutural cortical. Os demais casos desenvolveram ponte total. A obliteração da interface enxerto-osso hospedeiro foi evidenciada em 17 casos $(80,9 \%)$. Dezesseis pacientes $(76,2 \%)$ apresentaram reabsorção das arestas em grau variado, indicando remodelamento do fêmur.

Houve aumento do estoque ósseo em 90,5\% dos casos (19 pacientes), conforme valores do índice cortical proposto por Haddad et $a^{(3)}$, o que é corroborado por análise estatisticamente significante $(\mathrm{p}=0,001)$ entre as medidas radiográficas pré e pós-operatórias. O índice cortical médio encontrado no pós-operatório foi de 1,79, variando de 1,47 a 2,08. Essa aferição não pôde ser realizada em dois casos em virtude da presença de placa metálica.

A modificação da qualidade óssea femoral foi classificada como boa em $66,7 \%$ dos casos (14 pacientes) e ruim nos demais. A relação de melhora da qualidade óssea apresentou análise estatisticamente significativa ( $p=0,005$ para $5 \mathrm{~cm} \mathrm{e} \mathrm{p}=0,000$ para $10 \mathrm{~cm}$ ) entre as medidas radiográficas pré e pós-operatórias.

Houve três casos de alinhamento femoral não anatômico $(14,3 \%)$, apresentando varo de $5^{\circ}$ em dois casos e $10^{\circ}$ em um caso. Contudo, a alteração do alinha-

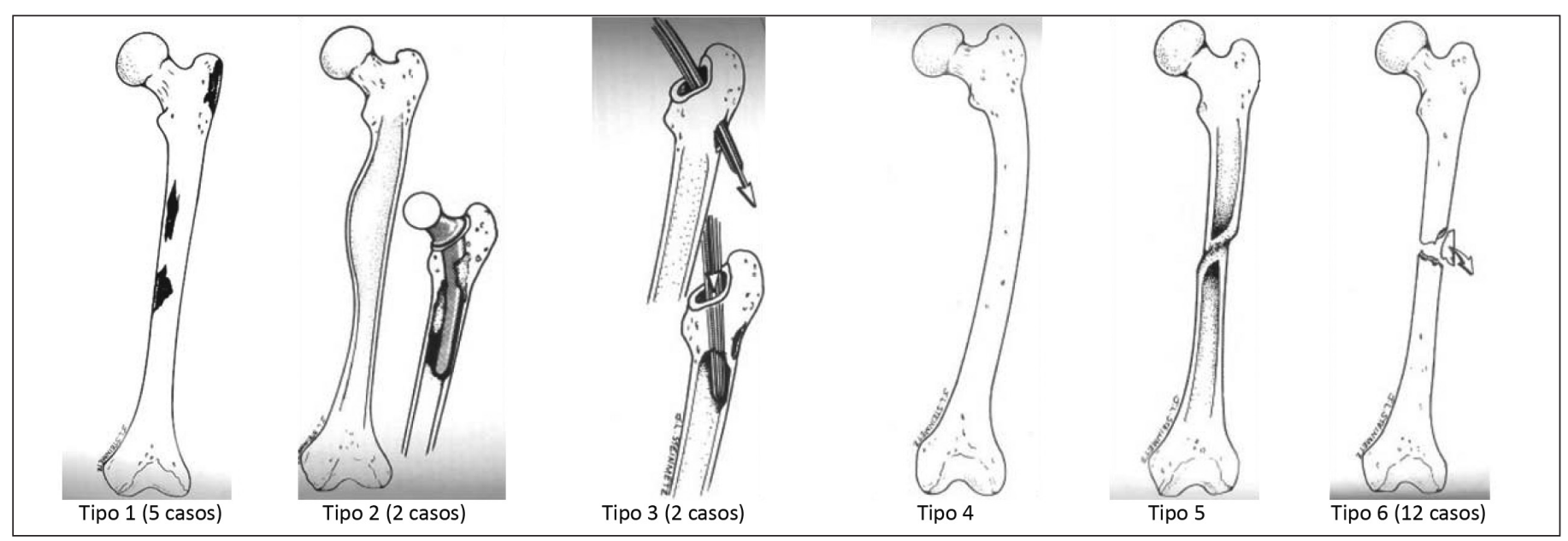

Figura 6 - Classificação de defeitos femorais da AAOS, segundo D'Antonio et a/ ${ }^{(19)}$. 
mento femoral não acarretou déficit funcional, pois o Harris Hip Score ${ }^{(17,18)}$ pós-operatório foi satisfatório.

Com relação às complicações, dois pacientes $(9,6 \%$ dos casos) apresentaram episódio de luxação do implante. Não houve casos de infecção.

\section{DISCUSSÃO}

$\mathrm{O}$ afrouxamento das artroplastias de quadril e as fraturas periprotéticas, frequentemente cursam com diminuição do estoque ósseo femoral, tornando seus tratamentos um desafio para os cirurgiões de quadril ${ }^{(1,2)}$. Existem diversas opções de tratamento relatadas na literatura, dentre as quais o uso de enxerto ósseo homólogo estrutural cortical, que tem se tornado um método atrativo em casos selecionados ${ }^{(3,13,16)}$.

Este tipo de enxerto pode agir como placa biológica, tanto isoladamente quanto de forma adjuvante em outras formas de fixação interna. Como o módulo de elasticidade do enxerto homólogo estrutural cortical é similar ao do osso hospedeiro, há menor estresse mecânico, se comparado a outras formas mais rígidas de fixação $0^{(3,22)}$.

Em estudos biomecânicos, Wilson et al ${ }^{(23)}$ compararam o estresse ósseo dos diferentes tipos de fixação para fraturas periprotéticas femorais, concluindo que a técnica do enxerto homólogo estrutural é uma excelente opção, pois melhora a estabilidade da fixação da fratura e aumenta o estoque ósseo.

Chandler ${ }^{(24)}$ e Chandler et al ${ }^{(25)}$ apresentaram o resultado do uso de enxerto ósseo homólogo estrutural no tratamento de fraturas periprotéticas de fêmur, com seguimento médio de 28 meses. No estudo com 19 casos de fraturas periprotéticas tratadas com redução aberta e fixação interna com enxerto ósseo homólogo estrutural cortical e cerclagem, obtiveram-se 16 casos $(84,2 \%)$ de união da fratura e avaliação funcional excelente.

Barden et $a l^{(16)}$ avaliaram 19 pacientes com fratura periprotética e significativa perda óssea tratados com enxerto ósseo homólogo estrutural em um seguimento médio de 3,7 anos. Houve consolidação em todos os casos e aumento do estoque ósseo femoral.

Haddad et $a l^{(3)}$ afirmam que os sinais radiográficos de consolidação óssea do enxerto homólogo estrutural cortical são vistos comumente antes do primeiro ano pós-operatório. Em uma série de 40 pacientes com fratura periprotética, 39 casos (98\%) apresentaram sinais radiográficos de consolidação antes do primeiro ano. Foi avaliada também a modificação do estoque ósseo por meio da análise radiográfica do índice cortical, observando-se melhora do mesmo.

A consolidação do enxerto homólogo estrutural cortical foi estudada por Emerson et al ${ }^{(13)}$ em revisões femorais de artroplastia total de quadril com perda de estoque ósseo. Foi observado um índice de $96,6 \%$ de consolidação do enxerto em um período médio de 8,4 meses, iniciando com pontes parciais, seguidas de pontes totais e do remodelamento do fêmur e do enxerto.

Callaghan et $a l^{(21)}$ avaliaram 92 pacientes submetidos à revisão femoral de artroplastia total de quadril com uso de enxerto ósseo homólogo estrutural em uma série com seguimento médio de 3,6 anos. Eles compararam a qualidade óssea em radiografias realizadas no préoperatório e pós-operatório tardio e classificaram-na como boa em $60 \%$ dos casos.

A escolha do seguimento mínimo de um ano como critério de inclusão ocorreu em virtude das constatações da literatura de que os sinais radiográficos de consolidação do enxerto estrutural cortical geralmente ocorrem no primeiro ano pós-operatório.

Uma das limitações do presente trabalho é o número restrito de pacientes incluídos na análise. Outra é a possível heterogeneicidade dos grupos de pacientes submetidos à reconstrução femoral secundária a afrouxamento de artroplastia e fratura periprotética.

No presente estudo foram constatados sinais radiográficos de consolidação do enxerto, tanto nos pacientes submetidos à reconstrução femoral secundária a afrouxamento de artroplastia quanto na reconstrução para fratura periprotética. Observou-se aumento do estoque ósseo em $90,5 \%$ dos casos e qualidade óssea do fêmur classificada como boa em $66,7 \%$ dos casos. Esses dados, somados aos achados clínicos satisfatórios dos pacientes, corroboram com os resultados da literatura de que a utilização de enxerto homólogo estrutural cortical é um importante método do arsenal terapêutico das reconstruções femorais com grande perda do estoque ósseo, bem como coadjuvante no tratamento da fratura periprotética.

\section{CONCLUSÃO}

O uso de enxerto ósseo homólogo estrutural cortical em cirurgias de reconstrução femoral de artroplastias totais do quadril e em fraturas periprotéticas é uma boa opção de tratamento em casos selecionados, permitindo resultados clínicos e radiográficos satisfatórios. 


\section{REFERÊNCIAS}

1. Head WC, Malinin TI, Emerson RH, Mallory TH. Restoration of bone stock in revision surgery of the femur. Int Orthop. 2000;24(1):9-14.

2. Haddad FS, Masri BA, Gasbuz DS, Duncan CD. Femoral bone loss in total hip arthroplasty: classification and preoperative planning. Instr Course Lect. 2000;49:83-96.

3. Haddad FS, Duncan CP, Berry DJ, Lewalen DG, Gross AE, Chandler HP. Periprosthetic femoral fractures around well-fixed implants: use of cortical onlay alografts with or without a plate. J Bone Joint Surg Am. 2002;84(6):945-50.

4. Lewallen DG, Berry DJ. Periprosthetic fracture of the femur after total hip arthrolplasty: treatment and results to date. Instr Course Lect. 1998;47:243-9.

5. Kelley SS. Periprosthetic femoral fractures. J Am Acad Orthop Surg. 1994;2(3):164-72

6. Brady $\mathrm{OH}$, Garbuz DS, Masri BA, Duncan CD. The treatment of periprosthetic fractures of the femur using cortical onlay alografts struts. Orthop Clin North Am. 1999;30(2):249-59.

7. Old AB, McGrory BJ, White RR, Babikian GM. Fixation of Vancouver B1 periprosthetic fractures by broad metal plates without the application of strut allografts. J Bone Joint Surg Br. 2006;88(11):1425-9.

8. Gie GA, Linder L, Ling RS, Simon JP, Slooff TJ, Timperley AJ. Impacted cancellous allografts and cement for revision total hip arthroplasty. J Bone Joint Surg Br. 1993;75(1):14-21.

9. Bono JV, McCarthy JC, Lee JA, Carangelo RJ, Turner RH. Fixation with a modular stem in revision total hip arthrplasty. Instr Course Lect. 2000;49:131-9.

10. Maloney WJ, Jasty M. Rosemberg A, Harris WH. Bone lysis in well-fixed cemented femoral components. J Bone Joint Surg Br. 1990;72(6):966-970.

11. Scott RD. Femoral fractures in conjunction with total hip replacement. J Bone Joint Surg Am. 1975;57(1):494-501.

12. Otte S, Fritzek J, Wedemeyer C, Löer F, von Knoch M, Saxler G. Reinforcement of deficient femur with inlay strut grafts in revision hip arthroplasty: a smal series. Arch Orthop Trauma Surg. 2006;126(10):649-53.

13. Emerson RH, Malinin TI, Cuellar AD, Head WC, Peters PC. Cortical strut alografts in the reconstruction of the femur in revision total hip arthroplasty: a basic science and clinical study. Clin Orthop Relat Res.1992;(285):35-44.

14. Drumond SN, Drumond FCF, Maranhão BKA, La Cruz LCR. Revisões femorais de artroplastias totais do quadril com afrouxamentos assépticos e fraturas periprotéticas: análise de 49 casos tratados com haste de Wagner. Rev Bras Ortop. 2007;42(7)206-16.

15. Crawford SA, Siney PD, Wroblewski BM. Revision of failed total hip arthroplasty with a proximal femoral modular cemented stem. J Bone Joint Surg Br. 2000;82(5):684-8

16. Barden B, von Knoch M, Fitzek JG, Löer F. Periprosthetic femoral fractures with extensive bone loss treated with onlay strut alografts. Int Orthop. 2003;27(3):164-7.

17. Harris WH. Traumatic arthritis of the hip after dislocation and acetabular fractures: treatment by mold arthroplasty - An end-result study using a new method of result evaluation.. J Bone Joint Surg Am. 1969;51(4):737-55.

18. Marchetti $P$, Binazzi R, Vaccari V, Girolami M, Morici F, Impallomeni C, et at. Long-term results with cementless Fitek (or Fitmore) cups. J Arthroplasty. 2005;20(6):730-7.

19. D'Antônio J, McCarthy JC, Bargar WL, Borden LS, Cappelo WN, Collis, DK et al. Classification of femoral abnormalities in total hip arthroplasty. Clin Orthop Relat Res. 1993;(296):133-9.

20. Masri BA, Meek RM, Duncan CP. Periprosthetic fractures evaluation and treatment. Clin Orthop Relat Res. 2004;(420):80-95.

21. Callaghan JJ, Salvati EA, Pellicci PM, Wilson PD, Ranawat CS. Results of revision for mechanical failure after cemented total hip replacement - 1979 to 1982. J Bone Joint Surg Am. 1985;67(7):1074-85.

22. Davidson D, Pikke, J, Garbuz D, Duncan CP, Masri BA. Intraoperative periprosthetic fractures during total hip arthroplasty. J Bone Joint Surg Am. 2008;90(9):2000-12.

23. Wilson D, Frei H, Masri BA, Oxland TR, Duncan CP. A biomechanical study comparing cortical onlay allograft struts and plates in the treatment of periprosthetic femoral fractures. Clin Biomech (Bristol, Avon). 2005;20(1):70-6.

24. Chandler HP. Reconstruction of major segmental loss of the proximal femur in revision total hip replacement. Orthopaedics. 1997;20(9):801-3.

25. Chandler HP, King D, Limbird R, Hedley A, McCarthy J, Penenberg B, et al. The use of cortical allograft struts for fixation of fractures associated with well-fixed total joint prostheses. Semin Arthroplasty. 1993;4(2):99-107. 